\title{
Central Bank Behaviour Concerning the Level of Bitcoin Regulation as a Policy Variable
}

\author{
By Beate Sauer*
}

\begin{abstract}
Bitcoin gains more and more attention in the general public and is already the most popular virtual currency. At the same time, the acceptance of Bitcoin as a speculative asset and also as a payment vehicle increases. This is an indication that we might now be entering an era of parallel currency systems. Therefore, one could state that the Bitcoin network and the central banking system could become two rival systems with respect to issuing payment vehicles and providing cross-border payment systems. Our aim is to analyse the central bank incentives for establishing a network model that includes hacking. With our model we are able to explain why central banks have no incentive to advance Bitcoin regulation at the current stage of development, as this would reduce the critical mass of Bitcoin users. Finally, in combination with a central bank loss function, we are able to calculate an optimal level of central regulation.
\end{abstract}

Keywords: Bitcoin, central bank, network theory, regulation, virtual currency

\section{Introduction}

The bitcoin gains more and more attention in the general public and it has evolved to be the most popular virtual currency, with a market capitalization of 3.9 billion $\mathrm{USD}^{1}$ in July 2015. At the same time, the acceptance of Bitcoin as a speculative asset and as a payment vehicle increases, reflecting the transition to an era of parallel currency systems. Therefore, it is quite possible that the Bitcoin network and the central banking system could enter a competitive market of issuing payment vehicles and providing cross-border payment options. In this context, central banks would normally not be interested in an advancement of the Bitcoin network and similar systems. Actually, they would rather have an incentive to work against the development of the Bitcoin network and to delay or even stop its growth. Evidence supporting this argument could be the lack of central bank publications on Bitcoin developments up till now. We concentrate on the Bitcoin, as it is the most popular representative of virtual currencies, albeit our scenarios and results can be extended to all other virtual currencies.

Our aim is to analyze central bank behaviour not only in a standard network model, but in the context of a model which includes hacking. In 2014, Mt. Gox, BTC-e, and Bitstamp, some of the largest Bitcoin exchange systems, recorded several successful hacking attacks, resulting in the bankruptcy of Mt. Gox. Therefore, it seems to be realistic to integrate hacking into the

\footnotetext{
* Postdoc and Lecturer, Bundeswehr University Munich, Germany.

${ }^{1}$ See http://coinmarketcap.com.
} 
network model. According to the review of the existing literature this is a novelty in Bitcoin network modelling. Also, we focus on central bank statements concerning Bitcoin, in an attempt to shed light on the reasons why the central banks mostly have a negative attitude towards Bitcoin. By using a standard network model, extended to include the option of hacking, we are able to integrate a very important policy variable of central banks in our baseline framework: the level of Bitcoin regulation, being discussed subsequently. Then, we argue that to treat the level of Bitcoin regulations as a policy variable seems to be adequate, as most of the central banks are involved in banking supervision and are also responsible for financial market stability. Finally, the results and conclusions are presented.

\section{Literature Overview}

Besides the original paper on virtual currencies from Nakamoto (2008), most of the Bitcoin literature focuses on the Bitcoin technology and the nature of the Bitcoin, as currency or commodity. Blundell-Wignall (2014) and Yermack (2013) give comprehensive overviews of the Bitcoin and its effects on economy, law, and taxation. Iavorschi (2013) concentrates on the comparison of the Bitcoin with natural money and Woo et al. (2013) calculates a fair value of the Bitcoin-USD exchange rate. Gomez-Gonzalez/Parra-Polania (2014) emphasise the high volatility of the Bitcoin price and its impact on the Bitcoin as a speculation asset, whereas Luther/White (2014) discuss the possibility of the Bitcoin becoming a major currency.

Iwamura et al. (2014) and Rogojanu/Badea (2014) draw parallels between the competition of virtual and national currencies based on Hayek's theory of competing currencies. Additionally, Gandal/Hałaburda (2014) control the network effects in their model and Bornholdt/Sneppen (2014) adapt a dynamited model. Malovic (2014), Arias/Shin (2013), and Hanley (2014) relativize the Bitcoin hype and express scepticism about the probability for it to become a widely accepted currency.

There are also a few survey articles from central banks (e.g. European Central Bank 2012, Bank of England 2014a and 2014b, Velde 2013) as well as from the financial institutions (e.g. European Banking Authority 2013) analysis about several issues associated to the use of the Bitcoin. Finally, there are also papers which focus on law and regulation of the Bitcoin (e.g. Plassaras 2013 or Global Legal Research Center 2014).

Issues like the role of the Bitcoin for portfolio diversification (Brière et al. 2013 and Dennis 2014), the characteristics of Bitcoin users (Wilson/Yelowitz 2014), money laundering (Stokes 2012) and modelling of Bitcoin' s interest rate dynamics (Wesner 2014) are more complicated and require a comprehensive analysis. So is the area of testing, evaluating and updating the existing network theory. For example, Frascatore/Mullen (2014) as well as Luther (2013) developed a model to analyse the network effect on Bitcoin adoption rates. 
As far as we know, one of the novelties in our model is the focus on the central bank behaviour concerning Bitcoin regulation. Central banks are very important players, being responsible for the stability of monetary and financial systems. Thus, they should be taken under consideration when modelling the Bitcoin network. Being based on our framework, we are able to conclude that central banks are not interested in the advancement of Bitcoin regulation at the current stage of the Bitcoin network.

A second novelty is the consideration of the possibility of hacking. In our opinion, hacking has to be included as it is closely connected to the Bitcoin network. The most famous hacking of Mt. Gox had significant influence on the Bitcoin price not only at that exchange (Figure 1). Even though the exchange rate collapse is a multidimensional phenomenon, it can be mainly explained from the perspective of a loss of trust in the Bitcoin network.

Figure 1. Bitcoin Price Development at Mt. Gox and BTC-e

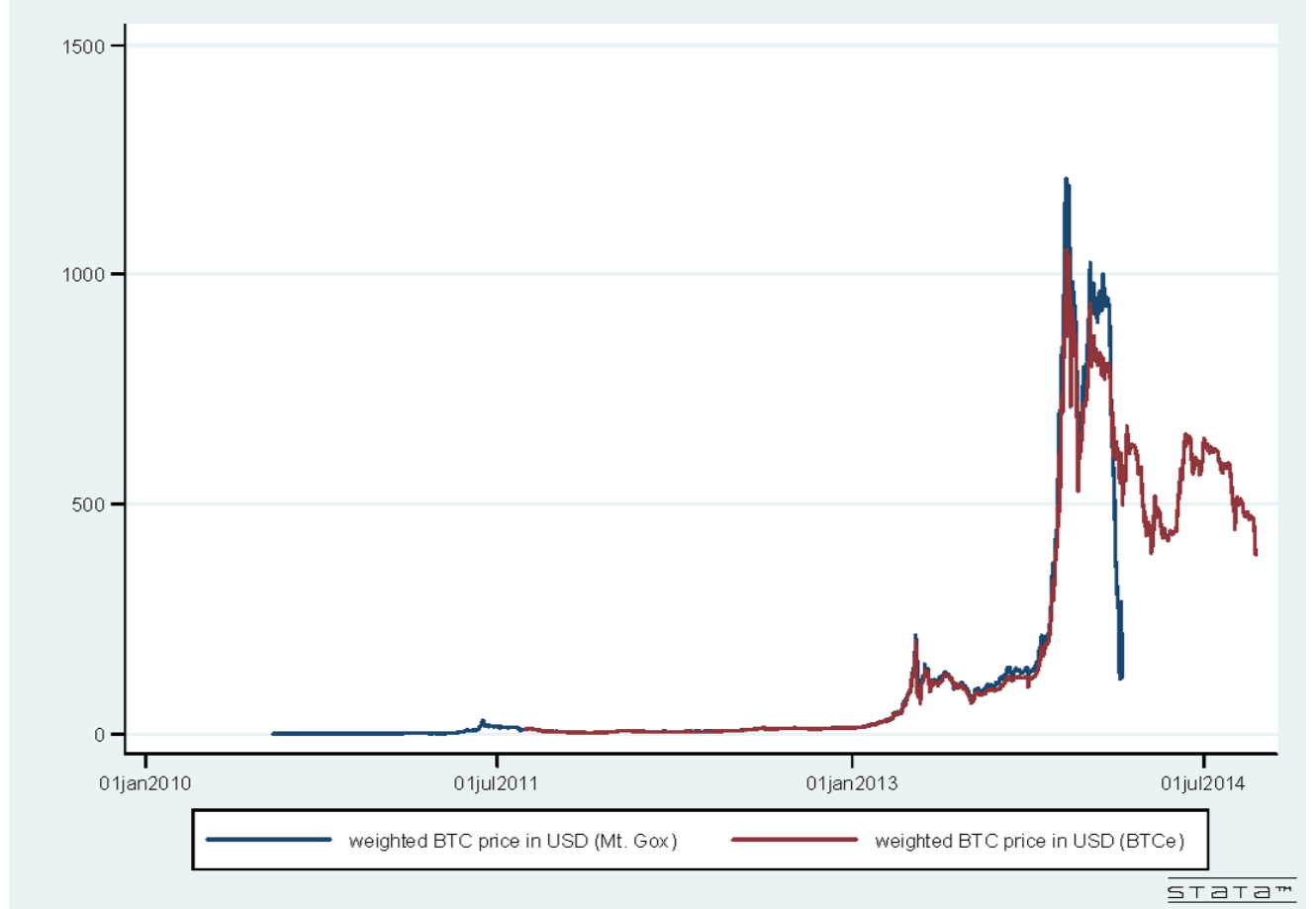

Source: http://bitcoincharts.com/charts.

\section{Central Banks and Bitcoin}

The Bitcoin was first introduced in 2008, thus, it is a quite young and totally new phenomenon: a decentralised virtual currency that can be used as a payment vehicle in real life. Thus, central banks and other financial authorities are faced with an unknown situation, threatening their money monopoly. This might be one explanation for the central banks' reserved attitude towards the growing Bitcoin community. A second explanation might lie in the ignorance 
over economic effects, especially on price and financial market stability, being the responsibility of central banks.

\section{Risks to Price Stability}

The Bitcoin's link to the real economy is already approved, as it can legally be used to buy and sell virtual and real goods and services. However, only a few major central banks have already published statements on the Bitcoin. The Bank of England and the ECB are two of them. Both are concerned that the Bitcoin would endanger general price stability. Following the ECB's statement (European Central Bank 2012: 34), virtual currencies, if used in real-economy transactions, might affect the economy's price levels, as they could modify the quantity of money and influence its velocity as well as the use of cash and the measurement of money aggregates.

Bitcoin mining is money creation, which means that Bitcoin supply has a direct positive impact on the overall supply of payment vehicles. If the Bitcoin partially substitutes the national currency, the central bank has to adapt its money supply to the reduced money demand, thus, resulting in a decline in overall national money supply.

Whether or not the Bitcoin influences the velocity of money depends on how fast the central bank reacts to money demand changes and how fast the Bitcoin is publicly accepted as a legit payment vehicle. If the use of cash effectively shrinks, a central bank balance sheet contraction is directly employed, constituting the first stage of the effect on the banking system. Consequently, the central bank loses part of its influence on the short term interest rates while the ECB even questions the functioning of the transmission mechanism (European Central Bank 2012). Also, the Bank of England assumes the extreme scenario that all day-to-day transactions are conducted in Bitcoin which would finally impair "the Bank's ability to influence price-setting and real activity ... severely" (Bank of England 2014a: 9).

\section{Risks to Financial Stability and to Payment System Stability}

Financial stability is mainly affected if the Bitcoin influences foreign exchange rates and the related expectations. Unfortunately, the share of the Bitcoin is very low which is why an empirical study on the forex market reactions is currently not feasible. The Bitcoin is a mainly non-regulated currency and is not controlled by any institution. Thus, the currency itself has to be seen as a risky asset and all those accepting Bitcoins have to be aware of this risk.

In the case where the majority of users gather significant computational power to intervene in transactions and to re-direct Bitcoin payment flows, a significant danger to the stability of the payment system could emerge which could even lead to a system-wide fraud. 


\section{Model Set-Up}

\section{Bitcoin Forex Market}

In our model the exchange rate is solely determined by speculators outside the network (Supply S Demand $_{s}$; Figure 2). The network users only use Bitcoin units as a payment vehicle and they do not speculate. This means that their buying and selling transactions correspond to each other on the forex market and do not change the exchange rate with the supply and demand curve shifting at the same level $\left(\right.$ Supply $_{S+U}$, Demand $\left.d_{S+U}\right)$. Hacking only reduces demand because of a loss of trust $\left(\right.$ Demand $\left._{h}\right)$. It is also assumed that hackers immediately sell the looted Bitcoins, leaving the supply constant, thus, resulting in a lower exchange rate. Hacking can only happen in the short time when the users act as buyers or sellers at the exchange market or in the case where their private computers get hacked. Besides, speculators react to changes in the expected exchange rate. An increase in the level of regulation increases the expected exchange rate, thus, resulting in enlarged rise in demand $\left(\right.$ Demand $\left._{h r}\right)$, a decline in supply $\left(\right.$ Supply $\left._{h r}\right)$ and a higher exchange rate.

Figure 2. Bitcoin Forex Market

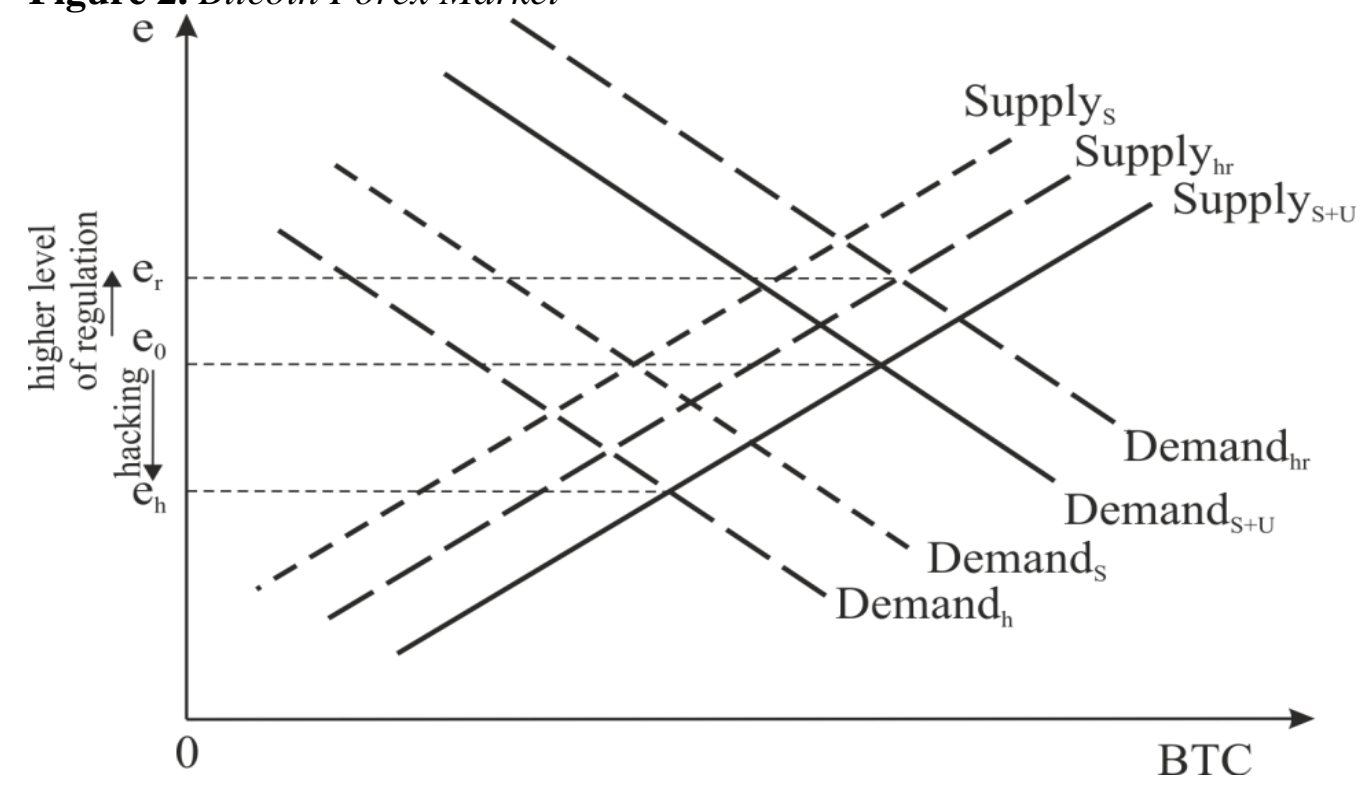

Network Model

Our network model is based on the model developed by Shy (2001: Ch. 5.2) and the extension with hackers, proposed by Bartholomae (2013). It consists of three players: the network user, deciding whether he will join the Bitcoin network (for transaction purposes) or not, the network hacker, with the decision of hacking a user/exchange or not, and the central bank as a representative of financial authorities having the option to impose regulation to transactions. 
Similar to Shy's analysis (2001) and with respect to the heterogeneity of users, the potential user group is defined as a $\eta$ continuum with a uniformly indexed preference $x \in[0,1]$ for the Bitcoin network and with density $\eta>0$. This implies that the higher the value of $x$, the lower the utility of the network for the user, being equivalent to low willingness to pay. Figure 3 visualises the density function and the cumulative distribution function.

Figure 3. Distribution of Potential Users

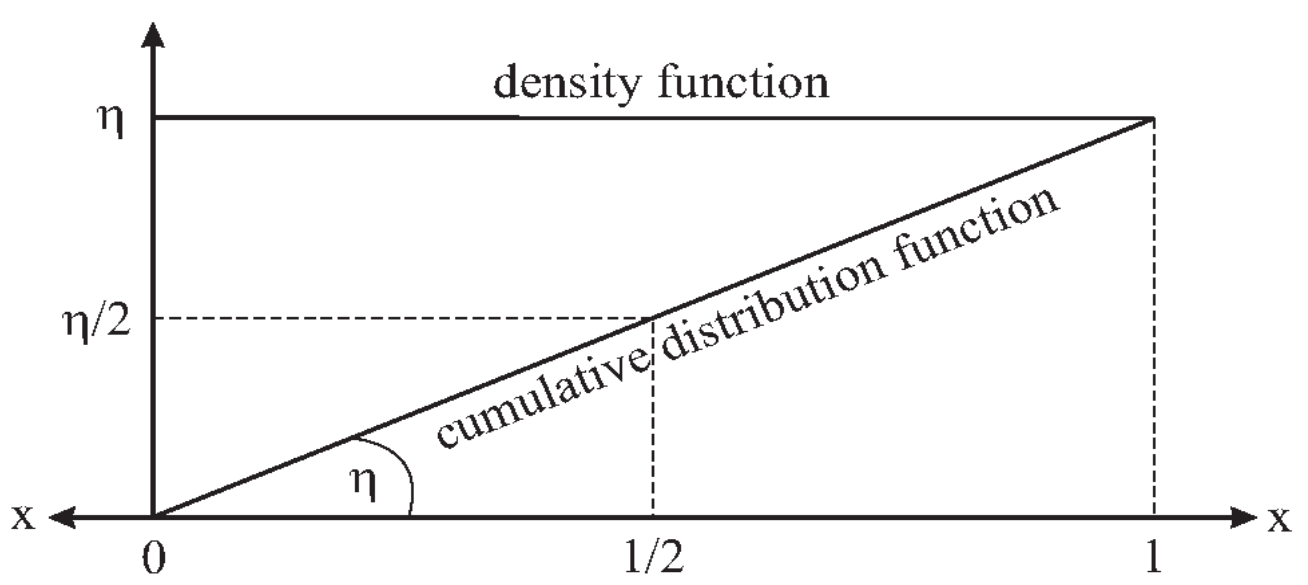

Source: Shy 2001: 110.

The user's expected utility, $U_{u}$, can be written as

$U_{u}=\left\{\begin{array}{lr}(1-x) \cdot N^{e}-p \quad \text { if joining the network, } \\ 0 \quad \text { if not joining the network, }\end{array}\right.$

Where $N^{e} \in[0, \infty)$ denotes the expected number of users and $p \in[0, \infty)$ represents the price of network access ${ }^{1}$. The indifferent user is

$$
(1-x) \cdot N^{e}-p=0 \quad \text { or } \quad x=\frac{N^{\theta}-p}{N^{e}}=: x_{u} .
$$

As $\frac{\partial x_{u}}{\partial N^{\varepsilon}}>0$, we can identify a positive network effect: with a higher expected number of users, $x_{u}$ increases, which means the indifferent user is now one with a lower willingness to pay. The overall number of users grows as more users join the network, even if some of those have actually lower willingness to pay (up to $x_{w}$ ). This seems to be logical as the more people that accept and use Bitcoin, the more attractive it gets. Consistent with Shy (2001) and Bartholomae (2013), we assume that users have perfect foresight to determine the expected and actual number of users: $N^{e}=N=\eta \cdot x_{u}$. Unfortunately, this is an unrealistic assumption, but it is an obligatory one for this model. Finally, for any $x$ the total network size is defined as $N=\eta \cdot x$.

\footnotetext{
${ }^{1}$ In contrast to Shy (2001) and Bartholomae (2013), the price is not a result of a firm's profit maximization, but is given by the cost of downloading software, a user fee of an exchange etc.
} 
The next stage is to introduce the hacker's decision with the utility $U_{h}$ :

$U_{h}=\left\{\begin{array}{lr}\chi \cdot \eta \cdot x \cdot b \cdot e+\alpha \cdot \eta \cdot x \cdot b \cdot e-\kappa \cdot S & \text { if hacking, } \\ 0 & \text { if not hacking, }\end{array}\right.$

With the first term representing the individual hacking, the second term standing for the exchange hacking and the third term defining the fine $(S>0)$ a hacker has to pay if he or she gets caught. The probability of this event is labelled $\kappa \in[0,1]$. The share of unprotected users in the Bitcoin network is $\chi$. These users are the only ones facing the danger of being directly hacked. The value of the user's data for the hacker is $b \cdot e$ (Bitcoin units $(b \geq 0)$ priced on the basis of the current exchange rate $(e \geq 0)$ ). If the hacker decides to hack an exchange, all exchange users lose the share $\alpha$ of the Bitcoin units they hold at that exchange. For reasons of simplicity, we assume that all users hold the same share of Bitcoins at a single exchange.

The hacker decides to hack if (see Bartholomae 2013: 6)

$\chi \cdot \eta \cdot x \cdot b \cdot e+\alpha \cdot \eta \cdot x \cdot b \cdot e-\kappa \cdot S>0$

and if $x>\frac{\kappa \cdot S}{(x+\alpha) \cdot \eta \cdot b \cdot e}$ with $\quad x=\frac{\kappa \cdot S}{(x+\alpha) \cdot \eta \cdot b \cdot e}=: x_{h^{a}}$

with $x_{h}$ describing "the user threshold that determines the network size in order to generate a positive expected net value of the network for the hacker" (Bartholomae 2013: 6). A higher fine increases the network size necessary to attract hackers. The same holds for the probability of a hacker being caught, $\kappa$. All remaining variables $(\chi, \alpha, b, e, \eta)$ have opposite effects.

The user's utility, $U_{u}$, can be extended with hacking to

$U_{u}=\left\{\begin{array}{lr}(1-x) \eta x-p-\chi b e+(\bar{r}-1) \alpha b e & \text { joining (hacking possible), } \\ 0 & \text { not joining, }\end{array}\right.$

With the joining decision only being taken when $x>x_{h}$. The damage of being directly hacked is the sum of the user's Bitcoin units priced with the current Bitcoin exchange rate multiplied by the share of unprotected users $(\chi b e)$. If exchanges are being hacked, the share of the Bitcoin units being held at that exchange rate times the current exchange rate $(\alpha b e)$ is lost. Depending on the given level of Bitcoin regulation, $\bar{r}$, part or all of the value of the stolen Bitcoins can be retrieved. The Bitcoin units themselves cannot be replaced as they are individual algorithms. If the private key is lost, the respective Bitcoin units are irrecoverable. In this context, Bitcoin regulation includes all kinds of statements and rules that ensure the system's reliability: questions on taxation, deposit insurance at exchanges, court rules, acceptance as equivalent currency etc. As a result, the financial loss that a user experiences when an exchange is being hacked decreases with advanced regulation $\left(\frac{\partial U_{u}}{\partial \bar{r}}>0\right)$. Total absence of regulation is reflected by $r=0$, whereas $r=1$ means that exchanges in the 
Bitcoin are totally regulated. The Bitcoin units which are held at private computers are not covered by this kind of protection for simplicity reasons.

A user decides to join the Bitcoin network if

$$
(1-x) \eta x-p-\chi b e+(\bar{r}-1) \alpha b e>0 .
$$

Solving this inequation for $e$ yields the inverse demand function for the Bitcoin network:

$$
e=\frac{p-(1-x) \eta x}{(\bar{r}-1) \alpha b-\chi b}
$$

The function shows two critical points, one at $x_{1}^{0}=\frac{1}{2}-\sqrt{\frac{1}{4}-\frac{p}{\eta}}$ and one at $x_{2}^{0}=\frac{1}{2}+\sqrt{\frac{1}{4}-\frac{p}{\eta}}$, with $e$ reaching its maximum at $x=\frac{1}{2}$. In the range $0 \leq x<\frac{1}{2}$ users join the network even if the network is small, meaning that the exchange rate effect dominates the network effect. In large networks $\left(\frac{1}{2}<x \leq 1\right)$ the network effect dominates the exchange rate effect and changes in inverse aggregate demand function are reflected in slope changes (analogously to Shy 2001: 112-13). As opposed to the specification of the demand function as proposed by Shy (2001), the whole function moves downwards because of the event of hacking. The two ranges $0 \leq x<x_{1}^{0}$ and $x_{2}^{0}<x \leq 1$ have to be eliminated because the inverse demand function only exists for positive exchange rates (Figure 4). Since a network needs at least two participants to exist, the starting point of the demand line is defined at $x_{1}^{0}$. The range $x_{2}^{0}<x \leq 1$ can be interpreted as follows: these users have such a low willingness to pay that they will never join the network. Users with network preference $x_{2}^{0}$ define the upper limit of the network size. It is argued that this modelling fits the Bitcoin network quite well as it seems to be unrealistic that everyone will join. 
Figure 4. Inverse Aggregate Demand Function

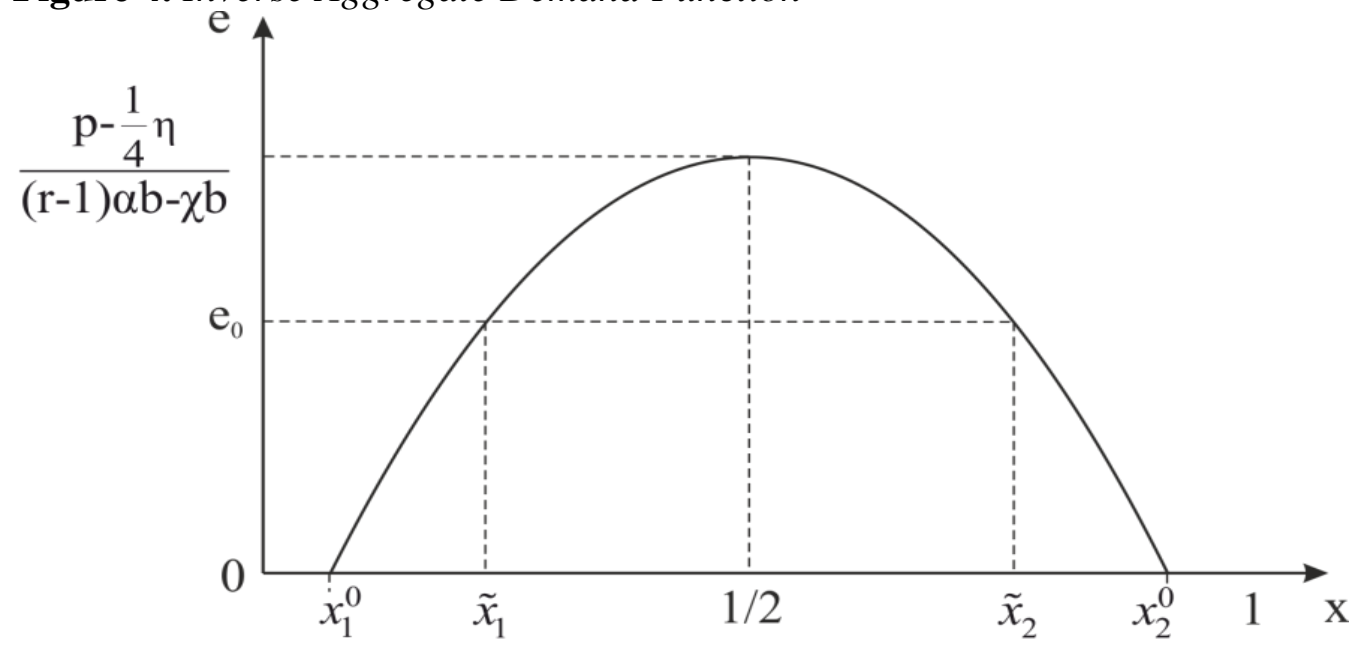

Source: Shy 2001: $112 \&$ Own adaptation.

A certain Bitcoin exchange rate $e_{0}$ (determined at the Bitcoin forex market) intersects the inverse demand function at the following points:

$\tilde{x}_{1}:=\frac{1}{2}-\sqrt{\frac{1}{4}+\frac{((\bar{r}-1) \alpha b-\chi b) \cdot \theta_{0}-p}{\eta}} \quad$ and $\quad \tilde{x}_{2}:=\frac{1}{2}+\sqrt{\frac{1}{4}+\frac{((\bar{r}-1) \alpha b-\chi b) \cdot \theta_{0}-p}{\eta}}$

According to Shy (2001: 112), convergence at the lower network size, $\tilde{x}_{1}$, is the critical mass for the network and hence an instable equilibrium. Namely, one user less makes the network even more instable while it could even lead to network failure. Naturally, one additional user makes the network more desirable and attracts all users with a preference for the network of $x>\tilde{x}_{1}$. This means that the larger the network size solution, $\tilde{x}_{2}$, the more stable the equilibrium.

Realistically, it is assumed that users' preferences extend to the range of $x_{1}^{0} \leq x \leq \frac{1}{2}$, where the exchange rate effect dominates the network effect and the network itself is not stable at all. Here, hacking reduces the network size (hacking $\rightarrow e \downarrow \rightarrow x \downarrow$ ).

\section{Central Bank Incentive and Optimal Policy}

As discussed above, the central banks and financial authorities appear to be interested in the Bitcoin network staying small or even failing, because the official systems and the Bitcoin network have conflicting interests. The following analysis focuses on a single central bank, even though any kind of Bitcoin regulation could result from a concerted action. 


\section{Incentive for Low Level of Bitcoin Regulation}

In order to identify the effect of the level of Bitcoin regulation on the critical mass of the network, we check the first deviation of $\tilde{x}_{1}$ :

$$
\frac{\partial \tilde{x}_{1}}{\partial r}=-\frac{\alpha b \theta_{0}}{2 \eta} \cdot\left(\frac{1}{4}+\frac{\left((\bar{r}-1) \alpha b-\chi^{b}\right) \cdot \epsilon_{0}-p}{\eta}\right)^{-\frac{1}{2}}<0
$$

The critical threshold of the network size is negatively influenced by the level of regulation, for a given exchange rate $e_{0}$. More precisely, a higher level of regulation would decrease the critical mass for the Bitcoin network and less regulation would increase the critical mass. If the network size stays or falls below $\tilde{x}_{1}$, the probability for a total network collapse is high.

The central bank has an incentive to increase the critical mass of the network or stop it from decreasing. This is possible by actively decreasing the level of regulation or at least by not advancing the regulation. For a central bank the best case would be to maximize $\tilde{x}_{1}$, which happens with $r=0$, letting the Bitcoin system to stay as unregulated as possible.

Because users take the exchange rate as given and react sensibly to official information like the level of regulation, the regulation effect on the critical mass outweighs the exchange rate effect; a lower level of regulation simultaneously decreases the exchange rate at the forex market via speculation, making a reduction in the critical mass possible.

\section{Incentive for High Level of Bitcoin Regulation}

Secondly, we analyse the effect of the regulation on a stable equilibrium. In this case, the Bitcoin network reaches a significant size and the network effect now outweighs the exchange rate effect. The first deviation reads as follows:

$$
\frac{\partial \tilde{x}_{2}}{\partial r}=\frac{\alpha b \varepsilon_{0}}{2 \eta} \cdot\left(\frac{1}{4}+\frac{((\bar{r}-1) \alpha b-\chi b) \cdot \epsilon_{0}-p}{\eta}\right)^{-\frac{1}{2}}>0
$$

As expected from the shape of the inverse demand curve, shown in Figure 4 , an increase in regulation would now increase $x$. Obviously, the central bank is able to hold the Bitcoin network instable for a longer time by implementing Bitcoin regulation. The maximum effect can be reached with setting $r=1$. The Bitcoin network would be totally regulated and controlled by supervisory authorities. Again, we have to take into account the users' behaviour of weighting the regulation effect more than the exchange rate effect.

\section{Central Bank’s Optimal Policy}

We now introduce the central bank's loss function, $L$, to in order to be able to solve the optimization problem. It includes the network size $(\eta x)$ multiplied 
by the squared users' utility and the cost of regulation minus the Bitcoin market capitalization multiplied by the level of regulation (ber), thus, capturing regulation utility. The loss function has the following form:

$L=\eta x \cdot\left(((1-x) \eta x-p-\chi b e+(r-1) \cdot \alpha b e)^{2}+r-b \cdot e \cdot r\right)$.

An increase in the users' utility is simultaneously a utility loss for the central bank. The latter is larger than the gain for the users because the central bank loses part of its own reputation as well as the reputation of the official currency system. Additionally, it may lose control over important variables like the interest rate or the inflation rate and this is why this term is squared in the above function. As the central bank is involved in financial market supervision, it is also involved in implementing any Bitcoin regulation, a costly procedure. The larger the network, the more effort it takes to introduce any kind of regulation, to control and to monitor the exchanges. Nevertheless, Bitcoin regulation is also useful for the central bank as it enables the partial control of the Bitcoin system and its incorporation into its sphere of responsibility. This is why the last term reduces the central bank's loss. At this point, we have to mention that we did not take into account the fact that central banks being involved in the Bitcoin network via regulation could also be negatively influenced by losing their reputation in the case of instable networks or hacking attacks.

Minimizing the loss function over $r$ gives:

$$
\frac{\partial L}{\partial r}=2 \eta x \cdot[(1-x) \eta x-p-\chi b e+(r-1) \alpha b e] \cdot \alpha b e+\eta x(1-b e)=0
$$

Solving for $r$ we get the optimal level of regulation:

$r^{*}=\frac{b_{\theta}-1-2 \alpha b_{\theta}\left[(1-x) \eta x-\chi b_{\theta}-\alpha b_{\theta}-p\right]}{2 \alpha^{2} b^{2} \theta^{2}}$,

which is a function of the network size $(\eta x)$. The first deviation shows us the direction of interdependence:

$$
\frac{\partial r^{*}}{\partial x}=\frac{\eta \cdot(2 x-1)}{\alpha b e}
$$

As can be seen in the numerator, an explicit result can only be found, when splitting the $x$-parameters at $x=\frac{1}{2}$. Depending on whether $x$ is less or greater than $\frac{1}{2}$, the central bank has a different optimal policy. In the first range, there is an incentive for a low level of regulation, as $\frac{\partial r^{*}}{\partial x}<0$ while in the second range, there is an incentive for a high level of regulation as $\frac{\partial r^{*}}{\partial x}>0$. In the case where Bitcoin users maximize their utility $\left(\frac{\partial U_{u}}{\partial x}=0\right)$, the optimal 
network size is reached exactly at $x=\frac{1}{2}$. The optimal level of regulation then takes the following form:

$r^{*}=\frac{b \theta-1-2 \alpha b e\left[\frac{\eta}{4}-x b^{b-}-\alpha b \theta-p\right]}{2 \alpha^{2} b^{2} e^{2}}$.

Under this certain condition the level of regulation is independent of the network size and the central bank finds itself between the extreme positions of total regulation or no regulation at all. In such a case, the central banks' decision is driven by other mechanisms. Realistically, one presumes these to be the exchange rate and the Bitcoin units, combined in the market capitalization. A central bank decision is not a binary decision. It has to take the current market situation into account, which is, inter alia, reflected in the exchange rate. For example, an increasing exchange rate increases $r$, thus regulation should be advanced.

At a first glance, the outcomes of the model seem to be in agreement with what has been happening in the market during the last two years. When the exchange rate was very high, official institutions started thinking about Bitcoin regulation. Bitcoin exchange rate and Bitcoin regulation seem to be positively correlated. But unfortunately, this specific prediction of this model describes the real trends, as it is unrealistic to assume that the optimal network size can be reached with $x=\frac{1}{2}$. The Bitcoin network is still in the beginning of its development while the central banks are certainly aware of the current situation of the Bitcoin network and its size. Therefore, they have no, or just a very low, incentive to impose regulation. On the other hand, it is a widely accepted fact that implementing any kind of regulation is not ad hoc. It needs time to discuss different proposals and even more time to pass a bill. Therefore, central banks manage to prepare regulatory laws or even implementing some soft ones, that do not reduce the critical mass of the network in a significant manner. Undoubtedly, they cannot only be observers of this kind of new development, but they also have to actively take part in this innovative area of the monetary system. Only being involved in the regulation of these systems, they might have the opportunity to benefit by some of the system's advantages in their own, official payment and financial systems and to control the unofficial ones.

With this explanation in mind the model's predictions for the first range succeeds to describe the current situation. This is indeed a good indicator that our model achieves to effectively highlight the importance of hacking for the Bitcoin network and to describe the possible central bank incentives concerning the level of Bitcoin regulation.

\section{Conclusions}

Bitcoin gains more and more attention not only in the general public, but also in the level of central banks and other financial authorities. From the view of central banks, the Bitcoin with its decentralized network structure takes the 
role of a rival, because it threatens their money monopoly and maybe also the stability of prices and of the financial system. As mentioned, this would imply that central banks may have an incentive to work against the development of the Bitcoin network. The various hacking incidents in the last months and the still relatively low regulation level contribute to the uncertainty of the Bitcoin network.

Due to the lack of comprehensive research on Bitcoin and the fact that many attempts to model the Bitcoin network on the basis of the existing network theories exist, we find it very challenging to use an adapted standard network model that would enable the integration of both hacking activities and the level of Bitcoin regulation.

Within our baseline framework, it is possible to study the central bank's incentives concerning the policy variable depending on the network size and also to calculate an optimal value for $r$. Central banks view the Bitcoin and its network with suspicion and presume it to be a rival in important fields of their responsibilities. Therefore, the main and realistic prediction is the incentive of central banks to not regulate the Bitcoin network at all. This policy will continue until the network reaches a size where the exchange rate effect is no longer dominant $\left(x>\frac{1}{2}\right)$. The optimal level of regulation depends no longer on the network size but on market capitalization, under the assumption of users' utility maximization. This level is located somewhere between the two extreme positions, as the central bank's decision is rarely binary, but rather multidimensional and flexible in order to react to unexpected events. However, as the Bitcoin is a decentralized currency, it needs central bank coordination to successfully implement any regulation.

\section{References}

Arias MA, Shin Y (2013) There are two Sides to Every Coin - Even to the Bitcoin, a Virtual Currency. The Regional Economist. Federal Reserve Bank of St. Louis. October.

Bank of England (2014a) The economics of digital currencies. Quarterly Bulletin. Q3. Retrieved from http://goo.gl/wJ6OAA.

Bank of England (2014b) Innovations in payment technologies and the emergence of digital currencies. Quarterly Bulletin. Q3. Retrieved from http://goo.gl/jnMaHu.

Bartholomae FW (2013) Network, Hackers, and Nonprotected Consumers. Working Papers in Economics 25(3). Bundeswehr University Munich.

Blundell-Wignall A (2014) The Bitcoin Question: Currency versus Trust-less Transfer Technology. OECD Working Papers on Finance, Insurance and Private Pensions. No. 37. OECD Publishing. Retrieved from http://goo.gl/ICIIZu.

Bornholdt S, Sneppen K (2014) Do Bitcoins make the world go round? On the dynamics of competing crypto-currencies. Cornell University Library. Ithaca, NY: arXiv. Retrieved from http://goo.gl/wZkL5c.

Brière M, Oosterlinck K, Szafarz A (2013) Virtual Currency, Tangible Return: Portfolio Diversification with Bitcoins. CEB Working Paper. No. 13/031. September. Université Libre de Bruxelles - Solvay Brussels School of Economics and Management, Centre Emile Bernheim. 
Dennis E (2014) Free Banking as a Monetary Gauge. Working Paper. Retrieved from http://goo.gl/1BmoHT.

European Banking Authority (2013) Warning to consumers on virtual currencies. EBA/WRG/2013/01.

European Central Bank (2012) Virtual Currency Schemes. October. Frankfurt a.M.

Frascatore MR, Mullen JK (2014) Heterogeneous Users and Adoption of Bitcoin: Adoption Rates, Network Effects, and Switching Costs. Paper prepared for presentation at the 77th International Atlantic Economic Society Conference in April. Madrid, Spain.

Gandal N, Hałaburda H (2014) Competition in the Cryptocurrency Market. Working Paper. No. 2014-33. Bank of Canada.

Global Legal Research Center (2014) Regulation of Bitcoin in Selected Jurisdictions. The Law Library of Congress: Washington, D.C.

Gomez-Gonzalez JE, Parra-Polania JA (2014) Bitcoin: something seems to be "fundamentally" wrong. Borradores de Economía. Banco de la Republica Colombia. No. 819.

Hanley B (2014) The false premises and promises of Bitcoin. Retrieved from http://goo.gl/RdxGiu.

Iavorschi M (2013) The Bitcoin Project and the Free Market. Centre for Economic Studies Working Paper Series 5(4): 529-534.

Iwamura M, Kitamura Y, Matsumoto T (2014) Is Bitcoin the Only Cryptocurrency in the Town? Economics of Cryptocurrency and Friedrich A. Hayek. Discussion Paper Series A. No. 602. Institute of Economic Research, Hitotsubashi University.

Luther WJ (2013) Cryptocurrencies, Network Effects, and Switching Costs. Working Paper. No. 13-17. Mercatus Center, George Mason University. September.

Luther WJ, White LH (2014) Can Bitcoin Become a Major Currency? Working Paper No. 14-17. Department of Economics, George Mason University.

Malovic M (2014) Demystifying Bitcoin: Sleight of Hand or Major Global Currency Alternative? Economic Analysis. 47(1-2): 32-41.

Nakamoto S (2008) Bitcoin: A Peer-to-Peer Electronic Cash System. Retrieved from https://goo.gl/MqyCW7.

Plassaras NA (2013) Regulating digital currencies: bringing bitcoin within the reach of IMF. Chicago Journal of International Law 14: 377.

Rogojanu A, Badea L (2014) The issue of competing currencies. Case study - Bitcoin. Theoretical and Applied Economics. Vol. XXI. No. 1(590). 103-114.

Shy O (2001) The Economics of Network Industries. Cambridge: Cambridge University Press.

Stokes R (2012) Virtual money laundering: the case of Bitcoin and the Linden dollar. Information \& Communications Technology Law 21(3): 221-236.

Velde FR (2013) Bitcoin: a primer. Essays on Issues. Chicago Fed Letter No. 317. The Federal Reserve Bank of Chicago.

Wesner N (2014) The Time Value of a Digital Currency: Bitcoin Interest Rates Dynamics. Retrieved from http://goo.gl/nlgx4t.

Wilson M, Yelowitz A (2014) Characteristics of Bitcoin Users: An Analysis of Google Search Data. MPRA Paper 59661. University Library of Munich, Germany.

Woo D, Gordon I, Iarolov V (2013) Bitcoin: A First Assessment. FX and Rates, Global. Bank of America. Merrill Lynch. December.

Yermack D (2013) Is Bitcoin a Real Currency? NBER Working Paper. No. 19747. Cambridge, MA. December. 